\title{
Oral Ursodeoxycholic Acid Crosses the Blood Retinal Barrier in Patients with Retinal Detachment and Protects Against Retinal Degeneration in an Ex Vivo Model
}

\author{
Alejandra Daruich ${ }^{1,2,3} \cdot$ Thara Jaworski $^{1} \cdot$ Hugues Henry ${ }^{4} \cdot$ Marta Zola $^{3} \cdot$ Jenny Youale ${ }^{1} \cdot$ Léa Parenti $^{1}$. \\ Marie-Christine Naud ${ }^{1} \cdot$ Kimberley Delaunay $^{1} \cdot$ Mathilde Bertrand $^{5} \cdot$ Marianne Berdugo $^{1} \cdot$ Laura Kowalczuk $^{3}$. \\ Jeffrey Boatright ${ }^{6,7}$ • Emilie Picard ${ }^{1}$ • Francine Behar-Cohen ${ }^{1,8}$ (D)
}

Accepted: 13 January 2021 / Published online: 3 February 2021

(C) The Author(s) 2021

\begin{abstract}
Rhegmatogenous retinal detachment (RD) is a threatening visual condition and a human disease model for retinal degenerations. Despite successful reattachment surgery, vision does not fully recover, due to subretinal fluid accumulation and subsequent photoreceptor cell death, through mechanisms that recapitulate those of retinal degenerative diseases. Hydrophilic bile acids are neuroprotective in animal models, but whether they can be used orally for retinal diseases is unknown. Ursodeoxycholic acid (UDCA) being approved for clinical use (e.g., in cholestasis), we have evaluated the ocular bioavailability of oral UDCA, administered to patients before RD surgery. The level of UDCA in ocular media correlated with the extent of blood retinal barrier disruption, evaluated by the extent of detachment and the albumin concentration in subretinal fluid. UDCA, at levels measured in ocular media, protected photoreceptors from apoptosis and necrosis in rat retinal explants, an ex vivo model of RD. The subretinal fluid from UDCA-treated patients, collected during surgery, significantly protected rat retinal explants from cell death, when compared to subretinal fluid from control patients. Pan-transcriptomic analysis of the retina showed that UDCA upregulated anti-apoptotic, anti-oxidant, and anti-inflammatory genes. Oral UDCA is a potential neuroprotective adjuvant therapy in RD and other retinal degenerative diseases and should be further evaluated in a clinical trial.
\end{abstract}

Keywords Retinal degeneration · retinal detachment · neuroprotection · ursodeoxycholic acid · UDCA · TUDCA

\section{Introduction}

The neural retina is made up of interneurons and photoreceptors, organized in several layers. The retinal photoreceptor cell

Emilie Picard and Francine Behar-Cohen contributed equally to this work.

Francine Behar-Cohen

francine.behar@gmail.com

1 Centre de Recherche des Cordeliers INSERM, UMRS1138, Team 17, Université de Paris, Université Sorbonne Paris Cité, Paris, France

2 Ophthalmology Department, Necker-Enfants Malades University Hospital, AP-HP, Paris, France

3 Ophthalmology Department, University of Lausanne, Jules-Gonin Eye Hospital, Fondation Asile des Aveugles, Lausanne, Switzerland

4 Innovation and Development Laboratory, Clinical Chemistry Service, Lausanne University Hospital, Lausanne, Switzerland layer is adjacent to the retinal pigment epithelium (RPE), a single-cell layer that provides support functions to the retina, recycles components critical to the visual cycle, and interfaces with the choroid, a vascular layer that provides metabolic

5 Institut du Cerveau et de la Moelle épinière (ICM), INSERM, CNRS, AP-HP, Sorbonne Université, Pitié-Salpêtrière University Hospital, Paris, France

6 Ophthalmology Department, Emory University School of Medicine, Atlanta, GA, USA

7 Center of Excellence, Atlanta Veterans Administration Medical Center, Decatur, GA, USA

8 Ophtalmopole, Cochin Hospital, AP-HP, Université de Paris, Paris, France 
support to the retina. The retina is attached to the RPE not only through structural factors such as its interaction with photoreceptor segments and extra cellular matrix but also by the active pumping mechanisms of the RPE. Rhegmatogenous retinal detachment (RRD) is a frequent form of retinal detachment (RD) that results from the mechanical tearing of the neural retina by vitreous tractions, leading to subretinal fluid (SRF) accumulation. It affects 13.3 per 100,000 habitants in Europe [1]. RD threatens vision, since photoreceptor cell death begins as early as $12 \mathrm{~h}$ after physical separation from the RPE and choroidal vessels [2]. Although reattachment surgery is performed urgently, only two-fifths of patients with RD involving the macula recover $20 / 40$ or better vision [3]. The irreversible loss of function is mainly due to photoreceptor cell death, through mechanisms that recapitulate those occurring in other degenerative retinal diseases [4]. RD is then a human model to test neuroprotective drugs.

A neuroprotective therapy adjuvant to surgery, targeting one or more mechanisms involved in photoreceptor cell death, could improve the visual outcome of patients operated on for RD.

Primary bile acids (BAs) are synthesized from cholesterol in the liver and then excreted into the intestine, where gut microbiota converts primary BA into secondary BA through chemical modifications. The main function of BA is the emulsification, absorption, and digestion of lipids.

However, the hydrophilic secondary BAs, ursodeoxycholic acid (UDCA) and tauroursodeoxycholic acid (TUDCA), the taurine conjugate of UDCA, have also shown neuroprotective effects in neurodegenerative [5] and retinal disease [6]. Recently, TUDCA showed beneficial effects in patients with amyotrophic lateral sclerosis in a randomized controlled clinical trial [5]. In the retina, TUDCA demonstrated anti-apoptotic and anti-oxidant effects in a rat model of RD [7], as well as in other models of photoreceptor degeneration, such as light-induced retinal damage and retinitis pigmentosa [8]. UDCA has shown beneficial effects on vascular integrity, inflammation, and endoplasmic reticulum stress in streptozotocin models of diabetic retinopathy $[9,10]$, but it has never been evaluated in RD models.

UDCA, unlike TUDCA, is FDA-approved for the treatment of cholesterol gallstone dissolution, and is currently considered the first-choice therapy for several forms of cholestatic syndromes [11]. Moreover, oral UDCA was shown to cross the blood-brain barrier in a dose-dependent manner with excellent tolerability and safety when administered in patients with amyotrophic lateral sclerosis [12]. The ocular bioavailability and tolerance of oral UDCA, however, has not been evaluated in patients with retinal pathology, which is a major limitation for its evaluation in retinal diseases. In the present study, we have measured UDCA levels in the ocular media and more specifically in the SRF of patients who were operated on for RD and treated with oral UDCA before surgery.
Next, we tested whether UDCA levels detected in SRF protected retinal explants from cell death, and we explored the mechanisms involved. Finally, we showed that, while SRF from untreated patients was toxic for rat retina explants, SRF from UDCA-treated patients was protective. Through the use of a reverse translational approach, we have brought forth evidence that oral UDCA could be used as an adjuvant therapy and improve retinal detachment surgery outcome.

\section{Methods}

\section{Clinical Study}

This study (ClinicalTrials.gov: NCT02841306) was designed in accordance with the tenets of the Declaration of Helsinki and was approved by the Ethics Committee of the Swiss Federal Department of Health (Authorization CER-VD no. 2016-00644). Written informed consent was received from all participants prior to inclusion. Twenty-six consecutive patients presenting with RRD for more than 4 days from symptom onset were included between the period of July 2016 and September 2016 at Jules-Gonin Eye Hospital, Lausanne, Switzerland. Exclusion criteria included the following: age $<18$ years or $>90$ years, monophthalmic patients, history of vitrectomy, vitreous bleeding or other associated retinal disease, and any condition or treatment contraindicating the administration of oral UDCA (pregnancy and lactation, peptic ulcer, acute or chronic liver disease, acute infection of the gallbladder and biliary tract, repeated biliary colic, Crohn's disease, ulcerative colitis, or other disease of the small intestine and colon, galactose intolerance, the Lapp lactase deficiency or glucose and galactose malabsorption, hypersensitivity, treatment by cholestyramine, colestipol, and antacids containing aluminum hydroxide or magnesium, cyclosporine, ciprofloxacin, nitrendipine, or dapsone). Baseline examination included a complete ophthalmological examination, ETDR assessment of best-corrected visual acuity (BCVA), and color fundus photography. Extension of RD was quantified as the number of clock hours of the fundus representation. Blood samples for UDCA determination $(400 \mu \mathrm{L})$ were collected at baseline (before surgery) and at day 7 after surgery, and were immediately centrifuged (twice at $2000 \mathrm{~g}$ ) for plasma extraction and congelation at $-80{ }^{\circ} \mathrm{C}$. Blood samples to test hepatic parameters (aspartate aminotransferase, AST (SGOT), alanine aminotransferase, ALT (SGPT), alkaline phosphatase ALP and gamma glutamyl-transpeptidase, $\gamma-$ GT) were collected at baseline, day 7, monthly for a period of 4 months, and at 6 months after surgery. Oral UDCA (Ursochol®, $300 \mathrm{mg}$, Zambon Switzerland Ltd., Cadempino, Switzerland) was administered at $10 \mathrm{mg} / \mathrm{kg} / \mathrm{day}$ before surgery at different intervals ( 4 groups: $\leq 5 \mathrm{~h}, 6-8 \mathrm{~h}, 9-11 \mathrm{~h}$, or $\geq 12 \mathrm{~h}$ ), and then after surgery (two-intake/day) during 
4 months. Five patients were not treated and served as control group. Standard $23 \mathrm{G}$ pars plana vitrectomy, laser or cryotherapy and gas or silicone tamponade, was performed in all patients. Ocular samples were collected during surgery before any surgical repair was undertaken, particularly cryotherapy. All surgeries and samples were performed by the same surgeon (AD) to minimize technical variability. Undiluted vitreous humor (VH) was collected via manual aspiration through a syringe connected to a 23-gauge vitrectomy cutter (before infusion opening; Alcon, Rotkreuz ZG, Switzerland). To collect SRF, the vitrectomy cutter was placed through the retinal tear under the retina before infusion opening, SRF was aspirated manually while infusion was slowly open to avoid hypotony and risk of bleeding. This procedure minimizes vitreous and infusion contamination. Samples were coded and stored in a biobank at $-80{ }^{\circ} \mathrm{C}$. Follow-up visits were performed at days 1 and 7, monthly for a period of 4 months, and at 6 months after surgery, and included a complete ophthalmological examination, color fundus photography, spectral-domain optical coherence tomography, microperimetry, and a questionnaire for patient-reported tolerance and occurrence of adverse events (Supplementary Table 1 and 2).

\section{UDCA and Protein Determination in Ocular and Plasma Samples}

Twenty-eight bile acids were measured by isotope-dilution high-performance liquid chromatography coupled to highresolution mass spectrometry (LC-MS HR). Each sample $(50 \mu \mathrm{L})$ was combined with the internal standards in methanol $(100 \mu \mathrm{L})$, and $\mathrm{H}_{2} \mathrm{O}$ with $0.2 \%$ formic acid $(600 \mu \mathrm{L})$ was added. Sample extraction and clean-up were performed using 96-well HLB SPE plates. Bile acids were separated via the method LC gradient within 22 min and were then measured by high-resolution MS. Quantification was based on peak area ratios interpolated against a seven-point calibration curve. The LC-MS HR system (QExactive Orbitrap mass spectrometer) was run using XCalibur 2.2 (Thermo Fisher Scientific, Cergy Pontoise, France). Data files were processed into result files using TraceFinder 3.0 (Thermo Fisher Scientific). Peak areas of target components were realized by auto-integration. The validated result file (TraceFinder 3.0) was exported in an Excel file format. Protein concentration in SRF was calculated using the Micro BCA protein assay (Thermo Fisher Scientific). Total protein concentration in SRF was calculated using the Micro BCA protein assay (Thermo Fisher Scientific).

\section{Cell Line and In Vitro Model}

WERI-Rb-1 human cone cell line (HTB-169, ATCC, Manassas, USA) were cultured on Roswell Park Memorial
Institute (RPMI)-1640 medium (Thermo Fisher Scientific) and supplemented with $10 \%$ fetal bovine serum and $1 \%$ penicillin-streptomycin. In order to determine the concentration of albumin needed to induce a significant cell death, WERI-Rb-1 human cones were treated with concentrations of albumin (Bovine Serum Albumin, Sigma-Aldrich Chemical Co., Saint-Quentin en Yvelines, France) from 2.5 to $40 \mathrm{mg} / \mathrm{mL}$ ( $n=4$ /group) and cultured during $24 \mathrm{~h}$. To determine the neuroprotective effect of UDCA (Sigma-Aldrich), WERI-Rb-1 human cones were treated by 1,5 , and $10 \mu \mathrm{M}$ of UDCA ( $n=4-8$ /group) $1 \mathrm{~h}$ earlier to add albumin at $20 \mathrm{mg} / \mathrm{mL}$. After $24 \mathrm{~h}$ of culture, $100 \mu \mathrm{L}$ of incubation medium was collected to determine cytotoxicity by lactate dehydrogenase (LDH) release (Sigma-Aldrich Chemical Co.), as previously described [13]. LDH activity in the incubation medium was compared with that measured after complete lysis of the cells in medium containing $2 \%$ Triton X-100. A viability percentage of zero corresponded to $100 \% \mathrm{LDH}$ activity in the medium. CellTiter (CellTiter 96® AQueous One Cell Proliferation Assay Solution, Promega Corporation, Charbonnières-LesBains, France) was used to assess mitochondrial activity. It was added to the cells and then incubated for $2 \mathrm{~h}$. The absorbance was read at $492 \mathrm{~nm}$. The results were calculated on percentage, by reporting the absorbance of cells by group to the mean of the control group.

\section{Animals and Ex Vivo Models of RD}

Adult male Wistar rats (Janvier labs, Le Genest St Isle, France, $n=59$ ) were fed with a standard laboratory diet and ad libitum tap water in a room maintained at $21^{\circ}$ to $23{ }^{\circ} \mathrm{C}$ with a $12-\mathrm{h}$ light/12-h dark cycle (6 a.m. to 6 p.m.) during 7 days, before being sacrificed by carbon dioxide inhalation, following French and European legislation (Décret no. 2013-118 du $1^{\mathrm{er}}$ Février 2013).

Retinal explants were created to mimic retinal detachment conditions. Neuroretinas were dissected from freshly enucleated eyes, separated from the retinal pigment epithelium, divided into two parts, and then transferred to 0.2-mm polycarbonate membranes (Millipore, Saint Quentin En Yvelines, France) with the photoreceptor layer facing up [13]. The membranes were next placed into a six-well culture plate containing Dulbecco's modified Eagle's medium (Thermo Fisher Scientific) and 3\% fetal bovine serum (3.9 ml/well). Three different models were used: spontaneous cell death (a model of retinal degeneration induced by retinal detachment), albumin-induced cell death, and patient SRF-induced cell death. First, in the spontaneous cell death model, $100 \mu \mathrm{L}$ of vehicle (control) or UDCA at a final concentration of 10, 50 (concentration rage found in patients), or $500 \mathrm{ng} / \mathrm{ml}$ (10 times these concentration range) was added to the photoreceptor side of the explants (upper chamber) ( $n=4 /$ group). Second, in the albumin-induced cell death model, $100 \mu \mathrm{L}$ of albumin 
(bovine serum albumin at $12 \mathrm{mg} / \mathrm{ml}$ ), alone (control) or containing UDCA at $10 \mathrm{ng} / \mathrm{ml}$ was added to the upper chamber ( $n=5 /$ group). And third, in the SRF-induced cell death model, $100 \mu \mathrm{L}$ of SRF from patients operated on in standard treatment of retinal detachment was added to the upper chamber. SRF from control $(n=5)$ and SRF from oral UDCA-treated patients (with UDCA found in SRF, $n=8$ ) were pooled before being added to the retinal explants. Five groups were compared: SRF from control patients $(n=9)$, SRF from control patients with addition of UDCA at $10 \mathrm{ng} / \mathrm{ml}(n=5)$, and SRF from oral UDCA-treated patients $(n=5)$.

The concentration of UDCA and albumin was chosen according to the concentration ranges found in the SRF of patients (except for the ten-time-dose of $500 \mathrm{ng} / \mathrm{ml}$ ), and serial dilution was performed to reach the final concentrations in the entire volume of the well $(4 \mathrm{ml})$. The dilutions of UDCA or vehicle were successively performed on ethanol $100 \%$ (10 mg/ml), phosphate-buffered saline (PBS), and medium (the same prepared for the culture wells). In the albumininduced cell death model, albumin was added at the last dilution. In the SRF-induced cell death model, the last two dilutions were performed directly within the SRF (and not in medium) (Supplementary Fig. 1). SRF from patients treated or not by oral UDCA was not diluted. Treated explants were cultured $48 \mathrm{~h}$ in spontaneous cell death model, and $6 \mathrm{~h}$ in the induced cell death models. LDH release was measured in medium collected from the lower chamber. Immunohistochemistry and Western blotting were performed on explants, as described below.

\section{Western Blotting Analysis}

Explants ( $n=4$ or 5 per group) were lysed MPER buffer (Thermo Fisher Scientific) and then centrifuged at 13,000g for $5 \mathrm{~min}$ at $4{ }^{\circ} \mathrm{C}$. Protein concentrations were calculated using the Micro BCA protein assay (Thermo Fisher Scientific). Five to $10 \mathrm{mg}$ of total extract was then mixed with protein loading buffer (Thermo Fisher Scientific), as per the manufacturer's instructions. Samples were loaded onto 4 to $12 \%$ bis-tris gel (Thermo Fisher Scientific), and proteins were transferred onto nitrocellulose membranes. Nonspecific binding was blocked with $5 \%$ nonfat dry milk in Tween/Tris-buffered saline, then membranes were incubated overnight at $4{ }^{\circ} \mathrm{C}$, with the primary antibody against Caspase 3 (1:500; Clone C92-605, BD Transduction Laboratories, CliniSciences, Nanterre, France), receptor-interacting protein (RIP) kinase 1 (1:500; Clone 38, BD Transduction Laboratories), apoptosis-inducing factor (AIF) (E-1) (1:500, Sc-13,116, Santa Cruz Biotechnology, Heidelberg, Germany), or actin (1:4000; Sigma-Aldrich), followed by incubation with the supplier-recommended dilution of horseradish peroxidase-conjugated secondary antibody for $1 \mathrm{~h}$ (Vector Laboratories, Eurobio, Les Ulis, France). Protein bands were visualized by an enhanced chemiluminescence reaction (Thermo Fisher Scientific) using a Bioimaging system (MicroChemi 4.2, Berthold, France or Invitrogen iBright Imaging Systems, Thermo Fisher Scientific). The gray values of specific bands were quantified using ImageJ, and the protein signals of interest were reported relative to the actin signal or to their respective cleaved forms (for Caspase 3, AIF) for each sample.

\section{Immunohistochemistry and Fluorescence Intensity Evaluation}

\section{Sections of Retinal Explants}

Explants were rinsed in $1 \times$ phosphate-buffered saline, fixed for 20 min with $4 \%$ paraformaldehyde (Inland Europe, Conflans sur Lanterne, France), infiltrated in a sucrose gradient series, and then mounted in Tissue-Tek optimum cutting temperature (OCT) (Siemens Medical, Puteaux, France). Immunohistochemistry was performed on 10- $\mu$ m-thick sections. Primary anti-rhodopsin antibody (Rho4D2, Abcam, Cambridge, UK) and the corresponding Alexa-conjugated secondary antibody (Thermo Fisher Scientific) were used; sections were counterstained with 4,6-diamidino-2phenylindole (DAPI, Sigma-Aldrich). Similarly, microglia and macrophages were immunodetected with ionized calcium-binding adapter molecule 1 (IBA1) (Wako Pure Chemical Industries, Neuss, Germany) staining. The sections were photographed with a fluorescence microscope (BX51, Olympus, Rungis, France), using identical exposure parameters for all compared samples. Blind quantifications were realized on photographs acquired at $\times 40$ magnification with ImageJ software. Measurement of the outer segment length was performed in 8 photographs/explant by measuring at three equidistant points on each photograph. The mean was then calculated for each explant ( $n=5$ explant/group). IBA1positive cells were quantified on photographs acquired at $\times 20$ magnification with ImageJ software, based on their differential shapes (round amoeboid or ramified dendritic form), as previously reported [14], and the results were expressed as a ratio (mean of 8 photographs/explant, $n=5$ explant/group).

\section{Flat-Mounted Retinal Explants}

Explants were rinsed in $\times 1$ phosphate-buffered saline, fixed for $20 \mathrm{~min}$ with $4 \%$ paraformaldehyde, and then permeabilized with $\mathrm{PBS} / 5 \%$ Triton $/ 5 \%$ fetal bovine serum for $45 \mathrm{~min}$. Incubation with Lectin from Arachis hypogaea (1/1000 Peanut Agglutinin, PNA-FITC, Sigma-Aldrich) was performed overnight at $4{ }^{\circ} \mathrm{C}$. DAPI (Sigma-Aldrich) staining was performed by incubation during $20 \mathrm{~min}$. Explants were flat-mounted between a slide and a coverslip, using a mounting medium (Fluoromount, Dako supplied by Agilent Technologies France, Les Ulis, France). The flat-mounted 
explants were photographed at $\times 40$ magnification with a fluorescence microscope (BX51, Olympus), using identical exposure parameters for all compared samples (6 photographs/explants). Blind quantifications of PNA-positive cells were realized on acquired photographs with ImageJ software.

\section{Real-Time Reverse Transcription Polymerase Chain Reaction}

Cell pellet ( $n=5$ per group) was directly frozen until ribonucleic acid (RNA) isolation with a RNeasy mini kit (Qiagen, Courtaboeuf, France), according to the manufacturer's protocols. RNA concentration and purity were determined with a NanoPhotometerTM (IMPLEN, Science Tec, Courtaboeuf, France). First-strand cDNA was generated through reverse transcription, using total RNA and a SuperScript IV Reverse Transcription Kit (Thermo Fischer Scientific). Quantitative PCR using duplicate technical replicates was performed on a Quant Studio 5 PCR System (Thermo Fischer Scientific) using SYBR Green gene expression assay probes (QuantiTech Primer Assay, Qiagen) of the human target genes sphingosine-1-phosphate receptor 2 (S1PR2, NM_004230; QT00230846), nuclear receptor subfamily 3, group C, member 1 (glucocorticoid receptor, GR) (NR3C1, NM_000176; QT00020608), nuclear receptor subfamily 3, group C, member 2 (mineralocorticoid receptor, MR) (NR3C2, NM_000901; QT000028490), g protein-coupled bile acid receptor 1 (GPBAR1, or Takeda $\mathrm{G}$ protein coupled receptor 5 (TGR5), NM_001077191; QT000209594), glyceraldehyde3-phosphate dehydrogenase (GAPDH, NM_001256799; QT00079247), and QuantiFast SYBR Green PCR Master Mix (Thermo Fischer Scientific). The expression levels of individual genes were then normalized with $G A P D H$ in the same sample by calculation of the $\Delta \mathrm{Ct}$ value, and relative quantification was performed using the $\Delta \Delta \mathrm{Ct}$ method, with condition without albumin and UDCA as control.

\section{Ribonucleic Acid Sequencing and RNA-Seq Data Analysis}

Retinas ( $n=3$ per group) were frozen immediately after isolation. Total RNA was extracted using a Precellys homogenizer (Bertin, Montigny-le-Bretonneux, France) and a RNeasy Mini kit (Qiagen). Quality of raw data has been evaluated with FastQC. Poor-quality sequences have been trimmed or removed with Trimmomatic software, in order to retain only good-quality paired reads. Star v2.5.3a has been used to align reads on reference genome using standard options. Quantification of gene and isoform abundances has been done with rsem 1.2.28 prior to normalization on library size with DESEq2 bioconductor package. Finally, differential analysis has been conducted with edgeR bioconductor package. Multiple hypothesis-adjusted $p$-values were calculated with the Benjamini-Hochberg procedure, to control FDR. Log2 fold-change threshold was set to 0.5 and the FDR threshold to 0.05 . At least $30 \%$ of samples per group respect a minimum CPM threshold of 1. Enrichment analysis has been performed with GSEA (Gene Set Enrichment analysis). Protein-protein interactions have been reconstructed with STRING (Search Tool for the Retrieval of Interacting Genes). The tool was used with a minimum required interaction score of 0.7 and the maximum number of interactors to show was set to 10 . The final network connecting the differentially expressed genes with the pathways was obtained with cytoscape.

\section{Statistics}

Results are presented as the mean \pm SEM. Analyses were performed using GraphPad (version 5.0, GraphPad Software, La Jolla, CA, USA). Non-normally distributed data was analyzed using a nonparametric Mann-Whitney test, in order to compare two groups. Spearman correlation coefficients were used to investigate association between variables. $p$ values $<0.05$ were considered statistically significant.

\section{Results}

\section{UDCA Level in Subretinal Fluid Correlates with Protein Concentration and with the Surface Area of the Detached Retina}

Twenty-six patients with RRD were included (mean age $=$ 62 years, male/female $=17 / 9$ ). All patients presented with simple RRD. Nineteen patients presented with macula off RRD and 7 patients with macula on RRD. Twenty-one patients received oral UDCA at different time intervals prior to surgery (including vitrectomy) for RD repair. Five patients who underwent surgery for RD, but did not receive UDCA, served as controls for ocular fluids (Supplementary Table 1). Ocular fluids, consisting of VH and SRF, were collected separately during surgery. Plasma samples were collected before UDCA was administered and at 7 days of treatment. While plasma UDCA concentration did not change significantly from baseline to 7 days in the control group (from $3.0 \pm 6.7$ to $15.3 \pm$ $27.0 \mathrm{ng} / \mathrm{ml}, p=0.6$, Mann-Whitney test), UDCA plasma levels increased significantly in the group treated with UDCA at 7 days, as compared to baseline (from $14.6 \pm$ 21.3 at baseline to $1363 \pm 1087 \mathrm{ng} / \mathrm{ml}$ at 7 days; $p<0.0001$, Mann-Whitney test) (Fig. 1(A)). UDCA concentration in plasma at 7 days was higher in UDCA-treated patients, compared with control patients $(1363 \pm 1087 \mathrm{ng} / \mathrm{ml}$ vs $15.3 \pm 27.0 \mathrm{ng} / \mathrm{ml}$, $p=0.0009$, Mann-Whitney test) (Fig. 1(A)). UDCA was measured in SRF or VH in 12 of the 21 treated patients ( $n=4 \mathrm{SRF}$, $n=4 \mathrm{VH}, n=4 \mathrm{SRF}$ and $\mathrm{VH})$. The mean concentration in $\mathrm{SRF}$ was $28.2 \pm 14.0 \mathrm{ng} / \mathrm{ml}(\mathrm{range}=12.9-49.0 \mathrm{ng} / \mathrm{ml})$, and 


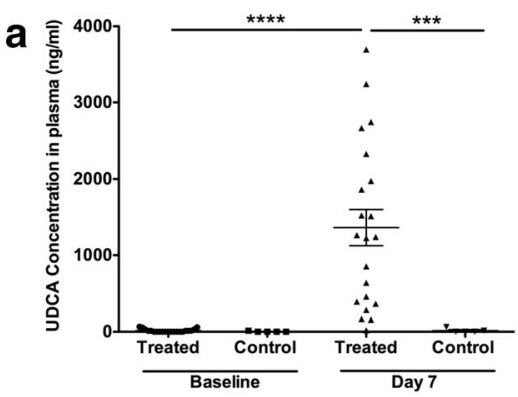

C

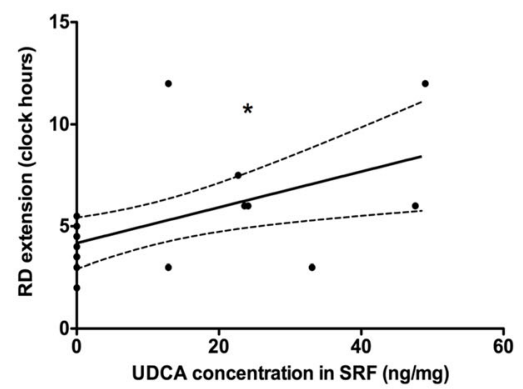

b

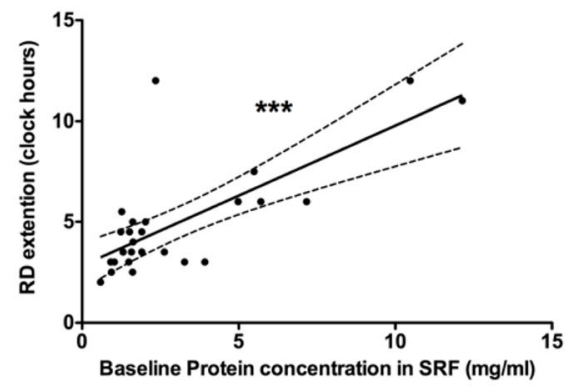

d

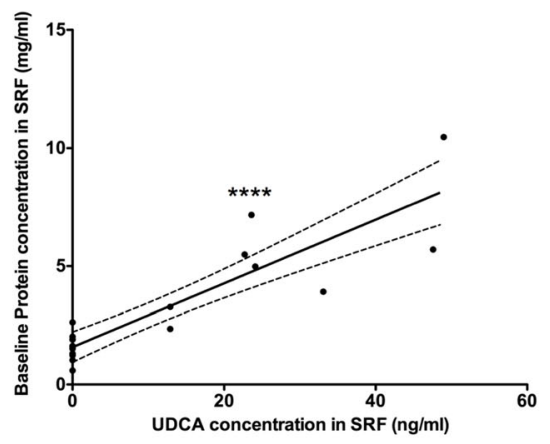

e
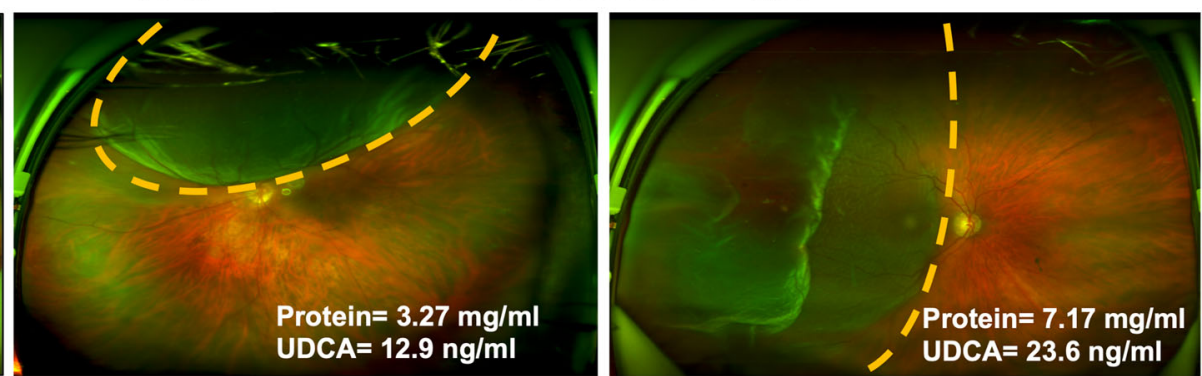

Fig. 1 UDCA concentration in plasma and ocular fluids relative to blood retinal barrier rupture in patients with retinal detachment (A) UDCA concentration in plasma at baseline, and at 7 days post-surgery in UDCA-treated patients $(n=21)$ and surgical controls not treated with UDCA $(n=5)$. UDCA concentration in plasma increased significantly only in treated patients at 7 days $(* * * * p<0.0001$, Mann-Whitney test). UDCA concentration in plasma at 7 days was significantly higher in UDCA-treated patients, when compared with control $(* * * p=0.0009$, Mann-Whitney test). (B) Baseline protein concentration in SRF was

in $\mathrm{VH}$, it was $19.6 \pm 9.9 \mathrm{ng} / \mathrm{ml}$ (range $=13.1-40.6 \mathrm{ng} / \mathrm{ml}$ ). UDCA was detected in one vitreous and in none of SRF of the 5 control patients. UDCA concentration in plasma at baseline did not correlate with the concentration of UDCA in ocular fluids ( $p=0.43, r=0.2$, Spearman correlation). The time interval between oral UDCA intake and ocular fluid sampling (range: 1.8-9.3 h) did not influence the concentration of UDCA in ocular fluids $(r=0.1, p=0.29$, Spearman correlation).

The extension of blood retinal barrier breakdown was evaluated by the surface area of detached retina at baseline expressed in the number of clock quadrants of the fundus representation and by the protein concentration in the SRF, that were both significantly correlated $(r=0.7, p=0.0002$, Spearman correlation) (Fig. 1(B)). Mean total protein concentration in SRF was $3.1 \pm 2.9 \mathrm{mg} / \mathrm{ml}$ (range: $0.6-12.1 \mathrm{mg} / \mathrm{ml}$ ). correlated with the extension of $\mathrm{RD}$ in clock hours at baseline $(p=$ $0.0002, r=0.7$, Spearman correlation). (C) UDCA concentration in SRF was correlated with the extension of retinal detachment (RD) in clock hours at baseline ${ }^{*} p=0.013, r=0.5$, Spearman correlation). (D) UDCA concentration in subretinal fluid (SRF) was correlated with protein concentration in SRF at baseline $(* * * * p<0.0001 ; r=0.8$, Spearman correlation). (E) Clinical examples showing the correlation between the extension of RD (dots line), the protein concentration in $\mathrm{SRF}$, and UDCA concentration in SRF

UDCA concentration in SRF significantly correlated with the surface area of detached retina $(r=0.5, p=$ 0.013 , Spearman correlation), and with protein concentration in SRF $(r=0.8, p<0.0001$, Spearman correlation) (Fig. 1(C-E)), demonstrating that the ocular bioavailability of UDCA is linked to the breakdown of the outer blood retinal barrier.

Clinical characteristics including change in BCVA at baseline and 6 months after surgery, UDCA levels, and proteins levels in treated and controls patients are reported in Supplementary Table 1 and Supplementary Fig. 2.

UDCA was well-tolerated, and few mild side effects (flatulence, constipation, unpleasant taste) were more frequently reported in UDCA-treated patients (Supplementary Table 2 and 3). Five of 21 patients interrupted treatment before 4 months had passed, due to nausea, epigastric pain, or 
tingling. Blood hepatic parameters remained within the normal ranges in all patients during the 6-month follow-up.

\section{UDCA, at Concentrations Found in Ocular Samples of RD Patients, Exerts Neuroprotective Effects in Rat Retinal Explants, an Ex Vivo Model of RD}

Rat neuroretinas, freshly separated from RPE and placed on membranes with photoreceptors facing up, mimic relevant pathological features of $\mathrm{RD}$ such as spontaneous photoreceptor cell death [13]. Rat retinal explants were treated with UDCA at concentrations detected in ocular fluids of treated patients $(10 \mathrm{ng} / \mathrm{ml}$, $50 \mathrm{ng} / \mathrm{ml}$ ), and at 10 times the highest concentration found in these patient ocular fluids (500 ng/ml), and cultured for $48 \mathrm{~h}$. Control rat retinal explants were treated with vehicle alone. $\mathrm{LDH}$, a cytosolic enzyme that is released early after cell membranes breakdown into the culture medium, was significantly lower in medium from retinas treated with UDCA at $10 \mathrm{ng} / \mathrm{ml}$ and $50 \mathrm{ng} / \mathrm{ml}$, compared to controls $(p=0.002$ and 0.046 , respectively, Mann-Whitney test), but not from those treated at $500 \mathrm{ng} / \mathrm{ml}$ (Fig. 2(A)). We used western immunoblotting in order to semi-quantitatively assess cell death pathway marker changes. The necrotic RIP kinase 1 was significantly reduced in retinas treated by UDCA at 10,50 , and $500 \mathrm{ng} / \mathrm{ml}$, as compared with control retinas ( $p=0.029$, Mann-Whitney test) (Fig. 2(B)). The apoptotic protein cleaved-Caspase 3 was significantly reduced in retinas treated by UDCA at $10 \mathrm{ng} / \mathrm{ml}(p=0.028$, Mann-Whitney test) and $50 \mathrm{ng} / \mathrm{ml}(p=0.014$, Mann-Whitney test), but not at $500 \mathrm{ng} / \mathrm{ml}$ (Fig. 2(C)). AIF, a Caspase-independent death effector [15], was significantly lower in retinas treated with UDCA at 10, 50 , and $500 \mathrm{ng} / \mathrm{ml}(p=0.029$, Mann-Whitney test), compared with control retinas (Fig. 2(D)). One of the early morphological markers in a detached neurosensory retina is the shortening of the outer segments of the photoreceptors. At the lowest concentration of $10 \mathrm{ng} / \mathrm{ml}$, UDCA significantly preserved photoreceptor morphology, as revealed by immunostaining on retinal sections and flat-mounted retinas. Rod outer segment length, labeled by rhodopsin (Rho4D2), was significantly higher in treated retinas, compared with control retinas $(p=0.029$, Mann-Whitney test) (Fig. 2(E)). In addition, the number of cone photoreceptor segments, specifically labeled on flat-mounted retinas with peanut agglutinin, was significantly higher in UDCA-treated retinas, compared to untreated retinas $(p=0.029$, Mann-Whitney test) (Fig. 2(F)).

\section{UDCA, at the Lowest Concentration Measured in Ocular Samples from RD Patients, Was Neuroprotective in Albumin-Exposed Rat Retina Explants}

As reported above, UDCA concentration in SRF was correlated with protein concentration in SRF (Fig. 1(C)). Thus, rat retinal explants were incubated for $6 \mathrm{~h}$ in medium containing albumin at the maximal protein concentration measured in the SRF of RRD patients $(12 \mathrm{mg} / \mathrm{ml})$ and compared to control explants treated with medium alone. A significant increase in LDH release was measured in the culture medium of rat neuroretinas exposed to albumin compared to control explants (Supplementary Fig. 3A). The effect of UDCA was then evaluated on rat retinal explants that were exposed to albumin alone (control) or albumin plus UDCA at $10 \mathrm{ng} / \mathrm{ml}$ for $6 \mathrm{~h}$. UDCA reduced LDH release significantly ( $p=0.04$, Mann-Whitney test), as compared to albumin exposure alone (Fig. 3(A)). RIP kinase was significantly lower in retinas treated with UDCA ( $p=0.02$, Mann-Whitney test), compared to those exposed to albumin alone (Fig. 3(B)), as well as cleaved-Caspase 3 ( $p=0.008$, Mann-Whitney test) (Fig. 3(C)), demonstrating that markers of both necrosis and apoptosis were significantly reduced in presence of UDCA at the concentration of $10 \mathrm{ng} / \mathrm{ml}$. Additionally, rod segment lengths labeled by Rho4D2 were significantly higher in UDCA-treated retinas compared with untreated retinas ( $p=0.01$, Mann-Whitney test) (Fig. 3(D)). Finally, the number of activated microglia cells (identified as ameboid cells) labeled by IBA1 was significantly reduced by UDCA treatment ( $p=0.008$, Mann-Whitney test) (Fig. 3(E)).

\section{UDCA Protected WERI-Rb-1 Human Cone-Like Cells from Albumin-Induced Death In Vitro}

Cone photoreceptor cells are most concentrated in the fovea and are responsible for high acuity vision. Therefore, we tested the effect of albumin on the WERI-Rb-1 human cone-like cell line. Albumin reduced cone viability in a dose-dependent manner (Supplementary Fig. 3B). At the concentration of $20 \mathrm{mg} / \mathrm{ml}$, albumin reduced by $40 \%$ cone cell viability after $24 \mathrm{~h}$ (Supplementary Fig. 3B). Prior to testing the direct effect of UDCA on WERI-Rb-1 cells, we showed that the bile acid receptors GR, MR, and TGR5 were expressed at the messenger RNA level. Pre-treatment with UDCA at the increasing concentrations of 1,5 , and $10 \mu \mathrm{M} 1 \mathrm{~h}$ before albumin exposure significantly increased the number of viable cells at 1 and $5 \mu \mathrm{M}(59 \% \pm 6$, mean increase of $11 \%, p=0.004$, and $59 \% \pm 3$, mean increase of $11 \%, p=0.01$, respectively, Mann-Whitney test, Fig. 3(F)). LDH release was significantly lower when cone cells had been previously treated with 1,5 , or $10 \mu \mathrm{M}$ of UDCA $(p=0.03$ for all concentrations, Mann-Whitney test, Fig. 3(G)).

\section{Effects of SRF Collected from Patients with RD on Rat Retinal Explants}

In order to more accurately mimic clinical conditions, rat retinal explants were incubated with a pool of SRF from RRD patients collected at the time of surgery. SRF from control patients (i.e., those not treated with UDCA) significantly increased markers of necrosis (LDH and RIP 1 kinase), as compared to explants cultured in medium alone (Supplementary Fig. $3 \mathrm{~A}$ and C). However, Caspase 3 cleavage was not 
a

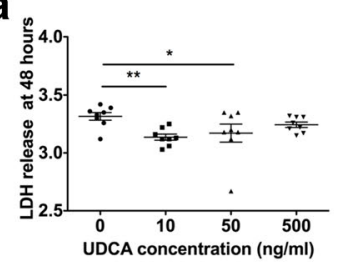

b
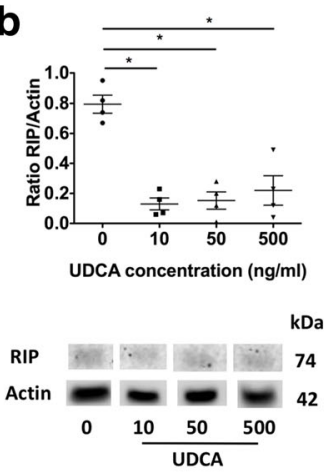

C
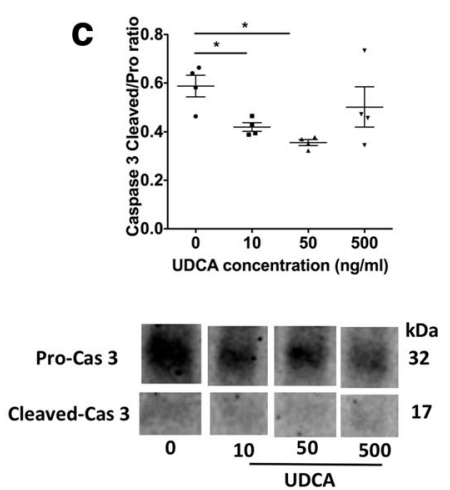

d
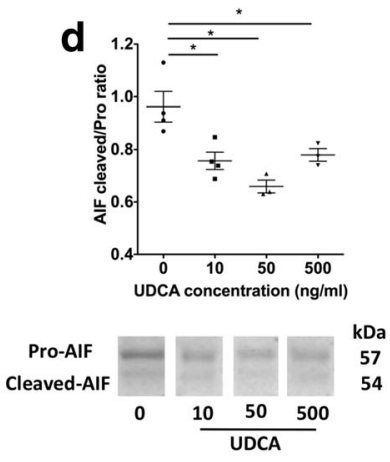

e
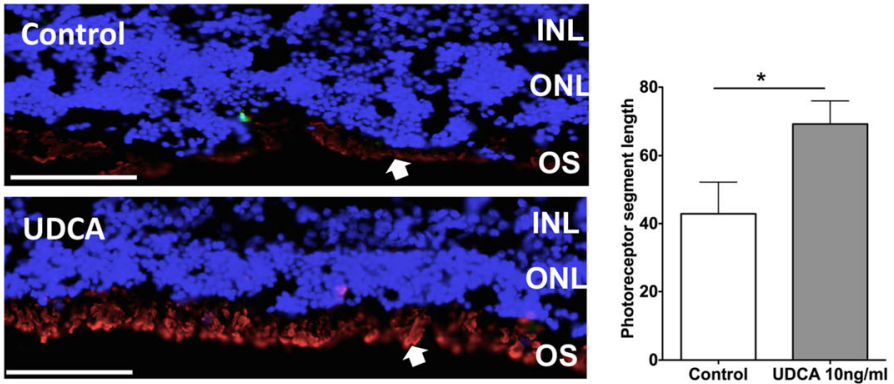

f
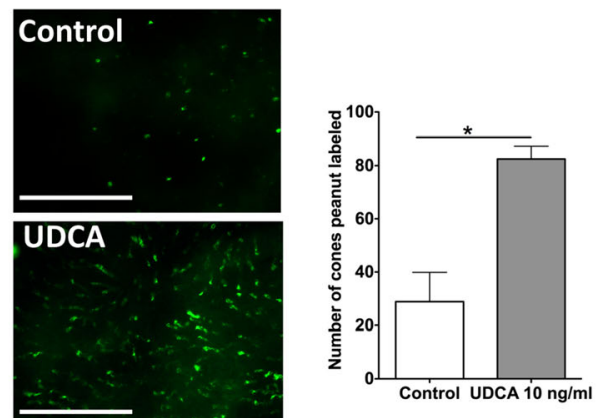

Fig. 2 UDCA protects from cell death and preserves photoreceptor in rat retinal explants. Retinal explants were treated with UDCA at concentrations found within patient ocular fluids $(10 \mathrm{ng} / \mathrm{ml}, 50 \mathrm{ng} / \mathrm{ml})$ and at 10 times the highest concentration $(500 \mathrm{ng} / \mathrm{ml})$ and cultured for $48 \mathrm{~h}$. Control explants were treated with vehicle. (A) Lactate dehydrogenase (LDH) release was lower in culture medium from retinas treated with UDCA at 10 and $50 \mathrm{ng} / \mathrm{ml}(* * p=0.002$ and $* p=$ 0.046, respectively). (B-D) Western blotting quantification. (B) Receptor-interacting protein (RIP) kinase reported on actin was reduced in UDCA-treated retinas $(* p=0.029)$. (C) The cleaved form of Caspase 3 reported on the full-form was reduced in retinas treated by UDCA at
$10 \mathrm{ng} / \mathrm{ml}\left({ }^{*} p=0.028\right)$ and $50 \mathrm{ng} / \mathrm{ml}(* p=0.014)$. (D) The cleaved form of apoptosis-inducing factor (AIF) reported on full form was lower in UDCA-treated retinas $(* p=0.029)$. (E) Immunostaining for Rho4D2 (red, arrows) in sections of retina explants. Control and UDCA (10 ng/ml) treated explants (left). Scale: $50 \mu \mathrm{m}$. Rod segments length was greater in treated retinas (right) $\left({ }^{*} p=0.029\right)$. (F) Immunostaining against cone segments (peanut agglutinin, green) on flat-mounted retina explants. Control and UDCA (10 ng/ml)-treated explants (left). Scale: $100 \mu \mathrm{m}$. Cone segments number was significantly higher in UDCAtreated retinas $(* p=0.029)$. Mann-Whitney test

found within the SRF from RD patients $(12 \mathrm{mg} / \mathrm{ml})$, and treated or not by UDCA. UDCA treatment significantly altered the expression of 38 genes; 31 were upregulated, and 7 were downregulated (Fig. 5(A)). Enriched analysis showed that hallmark gene sets were mostly implicated in apoptosis regulation, inflammatory response, oxidative stress, and neurogenesis (Fig. 5(B)and Supplementary Table 4), which could mediate the observed biologic effects. Genes upregulated by UDCA, such as Phldal, Gdf15, Tars, Asns, Pfkfb3, Chka, CD24, Hmox1, and Sphklencode proteins, involved in cell survival pathways, while Adcyapl, Atf3, Mt2A, Il4il, and Egrl and 2 encode proteins promoting neuroregeneration. $M y c, E d n 2$, Gadd45b, and Gadd45g encode proteins involved in both survival pathways in the retina and neuroregeneration. Hmoxl and Mt2A, encoding proteins with anti-oxidant properties, were also upregulated. On the other hand, UDCA also upregulated genes that were implicated in inflammatory response regulation, such as Il27, Il4i1, Atf3, Cebpb, Gdf15, Zfp36, Hmoxl, and Nr4A1. 
a
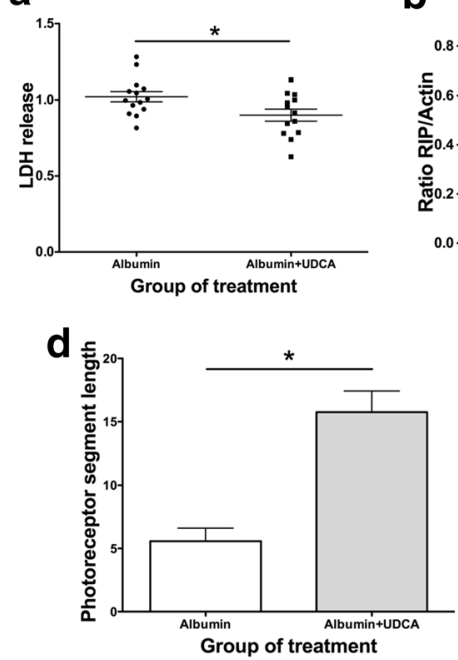

e

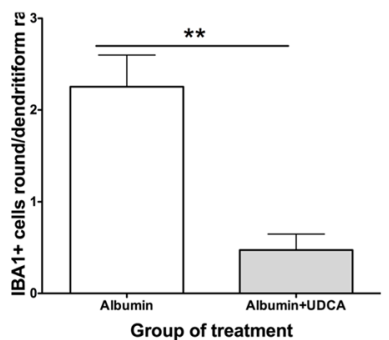

f

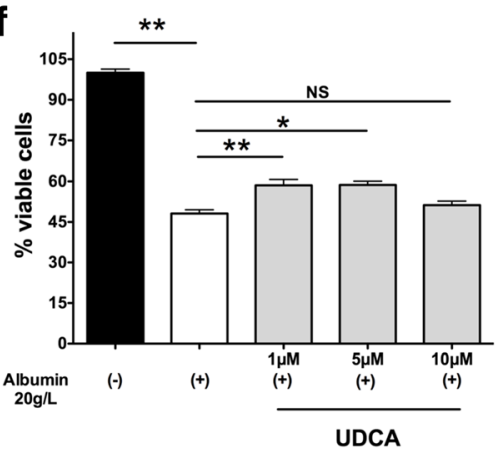

Fig. 3 UDCA protects from albumin-induced cell death ex vivo and in vitro. (A-E) Rat retina explants were treated by albumin or albumin and UDCA $10 \mathrm{ng} / \mathrm{ml}$ and cultured $6 \mathrm{~h}$. (A) Lactate dehydrogenase (LDH) release was lower in culture medium from retinas treated by UDCA $\left({ }^{*} p=\right.$ 0.04). (B-C) Western blotting quantification. Receptor-interacting protein $(\mathrm{RIP}) /$ actin ratio was lower in retinas treated by UDCA $(* p=$ $0.02)$ as well as $(\mathrm{C})$ cleaved/pro-Caspase 3 ratio $(* * p=0.008)$. (D-E) Immunostaining on sections of retinal explants. (D) Rod segments (Rho4D2, red, arrows) length was greater in UDCA-treated retinas

\section{Discussion}

UDCA, the first-line therapy for cholestatic liver diseases [11], has shown beneficial neuroprotective effects in various animal models of retinal diseases [6], but the ocular bioavailability of oral UDCA has not been previously evaluated, therefore limiting its clinical use. In this translational study, we demonstrated that in patients with retinal detachment, oral

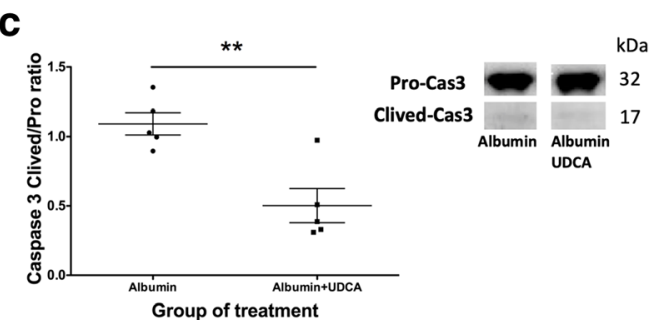

Group of treatment

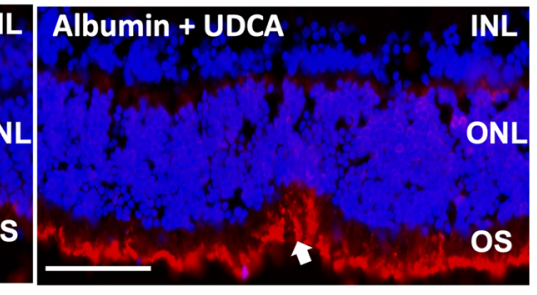

Albumin + UDCA

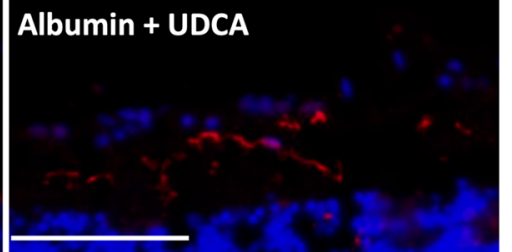

g

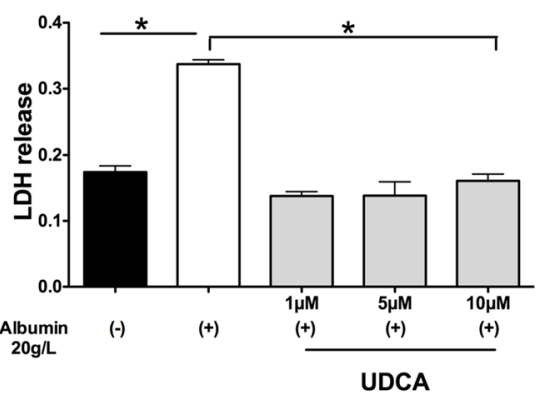

(* $p=0.01$ ). Scale: $50 \mu \mathrm{m}$. (E) Round/ramified ionized calcium-binding adapter molecule (IBA1)-positive cells (red) ratio was lower in UDCAtreated retinas $(* * p=0.008)$. Scale: $100 \mu \mathrm{m}$. $(\mathrm{F}-\mathrm{G})$ Human cones were treated by albumin and culture $24 \mathrm{~h}$. UDCA was added $1 \mathrm{~h}$ before albumin. (E) The $\%$ of viable cells decreased with albumin $(* * p=$ $0.001)$, but was higher when cones received 1 and $5 \mu \mathrm{M}$ of UDCA $(* * p=0.004$ and $* p=0.01)$. (F) Treatment by UDCA $(* p=0.03)$ decrease LDH release. Mann-Whitney test

UDCA reaches effective concentrations in the SRF. UDCA ocular bioavailability positively correlated with the surface area of the RRD and with the protein concentration in the SRF, hence suggesting that blood retinal barrier breakdown is the main factor that influences the ocular bioavailability of UDCA administered orally at $10 \mathrm{mg} / \mathrm{kg} /$ day. Surgical factors that alter the blood retinal barrier integrity, such as cryotherapy, could have influenced UDCA intraocular levels. 

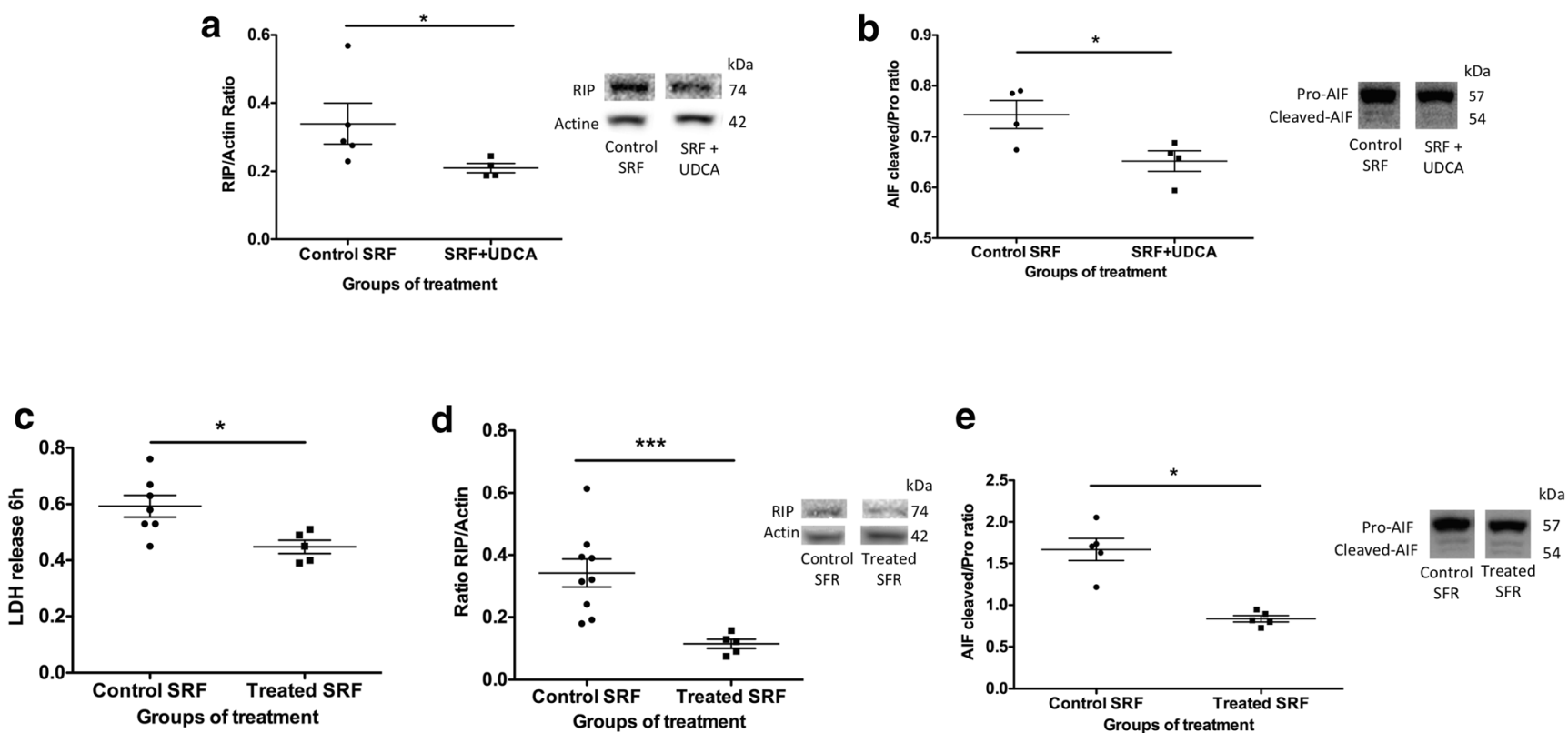

Fig. 4 UDCA protects from cell death in subretinal fluid (SRF)-exposed rat retina explants. (A, B) SRF from control patients with and without UDCA $(10 \mathrm{ng} / \mathrm{ml})$ was added to rat retina explants for $6 \mathrm{~h}$. (A, B) Western blotting quantification. (A) Receptor-interacting protein (RIP) kinase/ actin ratio was significantly lower in retinas treated by SRF + UDCA $\left({ }^{*} p=0.03\right)$ compared to control SRF. (B) Cleaved /pro-apoptosisinducing factor (AIF) ratio was significantly lower in retinas treated by $\mathrm{SRF}+\mathrm{UDCA}\left({ }^{*} p=0.03\right)$ compared to control SRF. (C-E) SRF from oral UDCA-treated patients and SRF from control patients were added to rat

retina explants for $6 \mathrm{~h}$. (C) Lactate dehydrogenase (LDH) release was lower in culture medium from retinas treated by SRF from oral UDCAtreated patients, compared to retinas treated by SRF from control patients $\left({ }^{*} p=0.02\right)$. (D, E) Western blotting quantification. (D) RIP/actin ratio was lower in retinas treated by SRF from UDCA-treated patients, compared to retinas treated by SRF from control patients $(* * * p=$ 0.001). (E) Cleaved/pro-AIF ratio was lower in retinas treated by SRF from UDCA-treated patients, compared to retinas treated by SRF from control patients $(* p=0.03)$. Mann-Whitney test

a

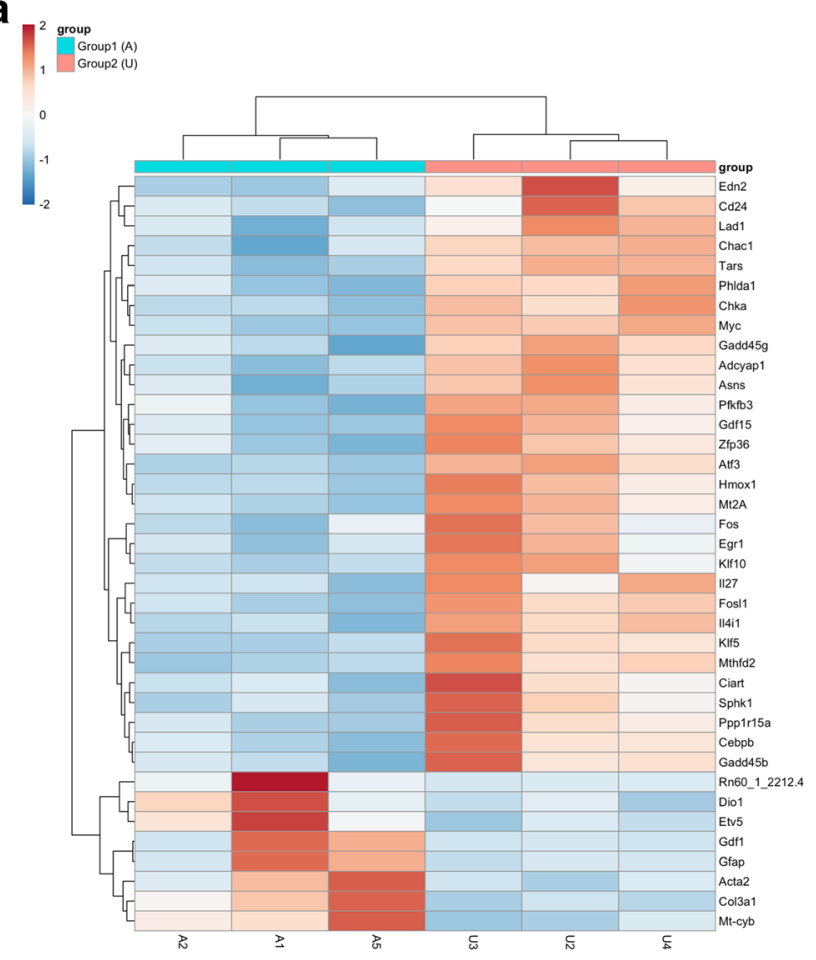

Fig. 5 RNA-seq analysis of rat retinal explants exposed to albumin alone or albumin + UDCA over 6 h. (A) Heatmap showed genes upregulated (red) and genes downregulated (blue) by albumin alone (A, group 1) b
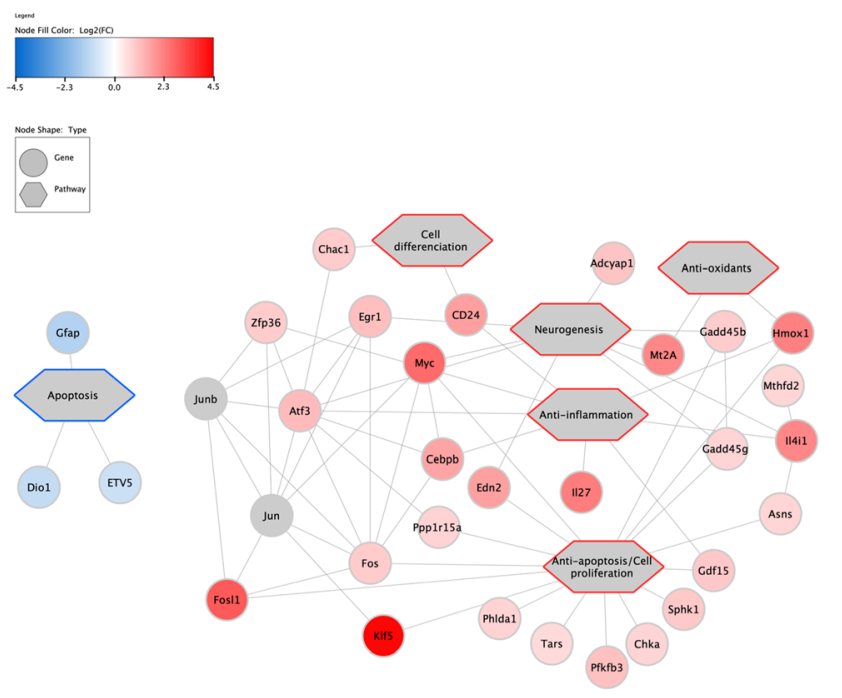

compared to UDCA plus albumin (U, group 2). (B) Network of the main genes upregulated (red) and downregulated (blue) by UDCA and the involved pathways 
However, in this study, all samples were taken before cryotherapy or any laser has been performed, excluding any interference with ocular biodisponibility of UDCA. These findings are consistent with observations in patients with amyotrophic lateral sclerosis $[5,12]$, a condition in which the blood-brain barrier is compromised [16]. In these patients, oral UDCA administered at doses ranging from 15 to $50 \mathrm{mg} / \mathrm{kg} /$ day crossed the blood-brain barrier in a dose-dependent manner. At the dosage of $10 \mathrm{mg} / \mathrm{kg} /$ day, the tolerance and safety of oral UDCA was confirmed in our study with only mild gastrointestinal tract adverse effects, as previously reported [12]. Finally, incubation with the SRF collected from patients and who had received oral UDCA reduced cell death in rat retinal explants, thereby suggesting that efficacious UDCA levels were reached in patients after oral administration. The correlation between visual outcome and ocular UDCA levels was not significant in this study that was not designed nor powered to show it. In addition, patients with higher UDCA levels were those with more extended RRD, and thus those expected to have worse visual outcome. But encouragingly, the later patients did not have worse visual outcome in this study. Only a randomized controlled study with clinically homogenous RRD patients could determine the functional and anatomical benefit of UDCA in patients with RRD. Whether UDCA reaches the eye mainly through the outer blood retinal barrier remains to be elucidated. At similar time duration between UDCA administration and ocular media sampling, UDCA was detected in vitreous, in SRF, or in both ocular fluids, while retinal levels could not be determined. UDCA concentration ranges in vitreous and SRF were similar which indicates the very low probability of infusion contamination. UDCA was measured in both compartments in 4 eyes and in one compartment in 8 eyes suggesting that low vitreous contamination occurred during SRF sampling. UDCA was measured in both compartments in 4 eyes and in one of the two compartments in 8 eyes suggesting that low vitreous contamination occurred during SRF sampling.

We thus believe that contamination of the LSR with vitreous or infusion fluid is very unlikely because the sampling was performed prior to any vitrectomy, limiting this risk as much as technically possible. But, if the SRF had been diluted with infusion fluid, we may have slightly underestimated the levels of UDCA in the SRF and may not have detected UDCA in some SRF. This would have led us to rather underestimate the passage of UDCA and therefore supports our results showing that oral administration could be used for patients with RD. In addition, in UDCA, being a hydrophobic compound, it is expected that retinal UDCA levels could be higher than ocular fluid ones. The passage of UDCA in the eye is probably controlled by many parameters, such as transporters, efflux proteins, and the integrity of the BRB. The fact that in untreated controls patients UDCA was measured in one single vitreous suggests that interindividual variability also controls the passage of endogenous UDCA into the eye.

Several approaches were implemented in exploring the possible benefit of UDCA and its mechanisms of action on cell death induced by retinal detachment. Incubation with UDCA at $10 \mathrm{ng} / \mathrm{ml}$ (a concentration measured in SRF of patients) protected retina explants from necrosis and caspasedependent and -independent cell death, as shown by the decreases of LDH release, RIP kinase activation, Caspase 3 cleavage, and AIF levels. At lower doses, UDCA also preserved the integrity of photoreceptor outer segments, which is recognized as a major visual prognosis factor in patients with RD [4]. The reason why higher doses of UDCA were not protective could potentially be related to the high hydrophobicity of the molecule, inducing direct interactions with the cell membrane, independently from receptor-mediated effects. Similarly, we also showed that glucocorticoids can exert toxic effects at higher doses, when in direct contact with the retina [17].

Various molecular components of SRF could exert toxicity on retinal neurons $[13,18]$ that, in physiologic conditions, are maintained by blood retinal barriers in a confined and controlled microenvironment. With increased RRD duration, SRF protein concentration increases [19] and visual prognosis worsens [20], but the direct effect of proteins on photoreceptors has not yet been evaluated. In the present study, protein concentration in SRF correlated with the detachment surface area. In addition, we showed that albumin reduced the viability of cultured immortalized human cone photoreceptors, suggesting a direct, toxic effect of albumin. Similarly, Liu et al. showed that cortical neurons are damaged by albumin uptake [21]. By adding albumin at the concentration measured in SRF from patients to retinal explants, in which the neuroretina is separated from the RPE, we intended to create an ex vivo $\mathrm{RD}$ model closer to the human clinical disease, in order to evaluate the neuroprotective properties of bile acids. Even in the presence of albumin, UDCA was still effective in reducing apoptosis and necrosis markers, as well as protecting photoreceptor integrity. However, the rat model has some limitations such as the absence of pigmented RPE and no macula. These results could thus be confirmed in retinal explants from other rodent strains, particularly pigmented ones as well as in human retina, although recent work showed that mechanisms of cell death in human retina explants are similar to those occurring in rat explants [22]. Since availability of human retinas is limited according to countries legislations, we tried to overcome the absence of fovea by additionally showing UDCA protection on human cone-like cells.

The protective effect of TUDCA was previously demonstrated against caspase activation in an in vivo RD model [7] and several models of photoreceptor degeneration [8], as well as against non-caspase-dependent mechanisms involving AIF 
in retinal cells exposed to glucose [23]. The exact contribution of taurine, the most abundant and neuroprotective amino acid in the retina [24] in these effects, had not been previously evaluated. Here, we showed that UDCA, which does not contain taurine, protected from apoptosis, and also from necrosis. When rat retinal explants were exposed to SRF from patients who had not received UDCA, caspase-dependent cell death activation was not observed, suggesting that SRF could have anti-apoptotic proprieties, and that caspase-dependent apoptosis may not occur in human RRD. In line with this hypothesis, although enzymatic activities of caspases have been reported in experimental models of RD [7], Caspase inhibition by panCaspase inhibitors failed to prevent photoreceptor loss [2].

Bile acid receptors have been described in the liver, intestine, and brain, and include nuclear receptors (Farnesoid $\mathrm{X}$ receptor (FXR), vitamin $\mathrm{D}$ receptor, pregnane $\mathrm{X}$ receptor, GR, and MR) and membrane receptors (TGR5, sphingosine 1-phosphate receptor 2, and $\alpha 5 \beta 1$ Integrin) [6]. Whether the beneficial effects of UDCA observed in the retina were mediated by these receptors is beyond the scope of this study. However, all of these receptors, with the exception of FXR, have been identified in the retina, and specific interactions have been reported for TUDCA and TGR5 in retinal ganglion cells [25].

UDCA altered the transcriptomic regulation of genes encoding proteins with anti-oxidant activities. Hmoxl, which encodes heme oxygenase-1, was significantly upregulated by UDCA, and is known to exert neuroprotection in the retina against oxidative stress injury [26], optical nerve section [27], and diabetic conditions [28]. In addition, the overexpression of heme oxygenase- 1 in a mouse model of RD protected photoreceptors from apoptosis [29]. Mt2A, which encodes metallothionein $2 \mathrm{~A}$ protein, protecting against hydroxyl free radicals and metal iron toxicity, preserved from various forms of retinal degeneration [30]. Atf3, upregulated by UDCA, is a member of the cAMP-response element binding protein family, which was upregulated in models of retinal and optic nerve regeneration [31], and a mutant Atf 3 mouse which showed reduced nerve regeneration [32]. Other genes upregulated by UDCA also encode proteins with potential neuroprotectant effects. Adcyap 1, which encodes pituitary adenylate cyclase-activating polypeptide (PACAP), was shown to carry neuroprotective properties in retinal degeneration and optic nerve crush models [33, 34]. Endothelin 2 showed protection of mutant photoreceptors in inherited photoreceptor degeneration [35], as well as protection against light damage [36]. $C d 24$ is a cell surface receptor expressed in photoreceptor precursors [37] and Chacl, which encodes a member of the gamma-glutamylcyclotransferase family of proteins, shown to promote neuronal differentiation and regulate glutathione levels [38]. Many genes involved in cell death were regulated, but more importantly, genes encoding anti-apoptotic proteins, such as Tars, Myc, Adcyapl, Asns,
Hmoxl, and Pfkfb3, and the anti-necrotic protein Hmoxl, were upregulated, identifying some of the potential mechanisms of action of UDCA. Additionally, Il27, which was upregulated, promotes the expression of the anti-oxidative protein complement factor $\mathrm{H}$, and reduces inflammation through IL10 expression in the retina [39, 40]. Through the release of pro-inflammatory cytokines [14], activated microglia are suspected to be neurotoxic to photoreceptor cells. Here, UDCA reduced the number of activated (ameboid) compared with normal (ramified) microglia, and we hypothesized that the observed normal microglia shape in UDCA-treated explants could be explained by the anti-inflammatory effects of UDCA. Altogether, these results indicate that, through transcriptional effects, UDCA regulates not only cell death pathways, but also retinal regeneration and inflammatory pathways.

In conclusion, this pilot study indicates that UDCA reaches the SRF proportionally to the extent of blood retinal barrier rupture. It reaches neuroprotective concentrations that efficiently reduce apoptosis and necrosis and preserve photoreceptor integrity. UDCA induces the expression of retinal genes with anti-oxidant, anti-apoptotic, and antiinflammatory properties. These results suggest that oral UDCA should be further evaluated clinically as adjuvant treatment to surgery in RD patients.

Supplementary Information The online version contains supplementary material available at https://doi.org/10.1007/s13311-021-01009-6.

Required Author Forms Disclosure forms provided by the authors are available with the online version of this article.

Funding This study was financially supported by Abraham J. and Phyllis Katz Foundation, USA; Fondation de 1'Avenir, France; Association Française des Amblyopes Unilatéraux, France; Prix Retina 2017, Swiss VitreoRetinal Group and Novartis, Switzerland; and NIH R01EY028859, R01EY028450, P30EY06360, and VA I01RX002806 and I21RX001924, VARR\&D C9246C USA.

\section{Declarations}

Conflict of Interest The authors declare no competing interests.

Disclaimer The sponsors did not have any role in the study.

Open Access This article is licensed under a Creative Commons Attribution 4.0 International License, which permits use, sharing, adaptation, distribution and reproduction in any medium or format, as long as you give appropriate credit to the original author(s) and the source, provide a link to the Creative Commons licence, and indicate if changes were made. The images or other third party material in this article are included in the article's Creative Commons licence, unless indicated otherwise in a credit line to the material. If material is not included in the article's Creative Commons licence and your intended use is not permitted by statutory regulation or exceeds the permitted use, you will need to obtain permission directly from the copyright holder. To view a copy of this licence, visit http://creativecommons.org/licenses/by/4.0/. 


\section{References}

1. Li JQ, Welchowski T, Schmid M, Holz FG, Finger RP. Incidence of Rhegmatogenous Retinal Detachment in Europe - A Systematic Review and Meta-Analysis. Ophthalmologica 2019:1-6. https:// doi.org/10.1159/000499489.

2. Murakami Y, Notomi S, Hisatomi T, Nakazawa T, Ishibashi T, Miller JW, et al. Photoreceptor cell death and rescue in retinal detachment and degenerations. Prog Retin Eye Res 2013;37:114 40. https://doi.org/10.1016/j.preteyeres.2013.08.001.

3. Campo RV, Sipperley JO, Sneed SR, Park DW, Dugel PU, Jacobsen J, et al. Pars plana vitrectomy without scleral buckle for pseudophakic retinal detachments. Ophthalmology 1999;106: 1811-5; discussion 1816. https://doi.org/10.1016/S0161-6420(99) 90353-6.

4. Matsui A, Toshida H, Honda R, Seto T, Ohta T, Murakami A. Preoperative and Postoperative Optical Coherence Tomography Findings in Patients with Rhegmatogenous Retinal Detachment Involving the Macular Region. ISRN Ophthalmol 2013;2013:1-6. https://doi.org/10.1155/2013/426867.

5. Paganoni S, Macklin EA, Hendrix S, Berry JD, Elliott MA, Maiser $\mathrm{S}$, et al. Trial of Sodium Phenylbutyrate-Taurursodiol for Amyotrophic Lateral Sclerosis. N Engl J Med 2020;383:919-30. https://doi.org/10.1056/NEJMoa1916945.

6. Daruich A, Picard E, Boatright JH, Behar-Cohen F. The bile acids urso- and tauroursodeoxycholic acid as neuroprotective therapies in retinal disease. Mol Vis 2019.

7. Mantopoulos D, Murakami Y, Comander J, Thanos A, Roh M, Miller JW, et al. Tauroursodeoxycholic acid (TUDCA) protects photoreceptors from cell death after experimental retinal detachment. PLoS One 2011;6:e24245. https://doi.org/10.1371/journal. pone.0024245.

8. Boatright JH, Moring AG, McElroy C, Phillips MJ, Do VT, Chang $\mathrm{B}$, et al. Tool from ancient pharmacopoeia prevents vision loss. Mol Vis 2006;12:1706-14.

9. Chung Y-R, Choi JA, Koh J-Y, Yoon YH. Ursodeoxycholic Acid Attenuates Endoplasmic Reticulum Stress-Related Retinal Pericyte Loss in Streptozotocin-Induced Diabetic Mice. J Diabetes Res 2017;2017:1763292. https://doi.org/10.1155/2017/1763292.

10. Ouyang H, Mei X, Zhang T, Lu B, Ji L. Ursodeoxycholic acid ameliorates diabetic retinopathy via reducing retinal inflammation and reversing the breakdown of blood-retinal barrier. Eur $\mathrm{J}$ Pharmacol 2018;840:20-7. https://doi.org/10.1016/j.ejphar.2018. 09.027.

11. Wagner M, Fickert P. Drug Therapies for Chronic Cholestatic Liver Diseases. Annu Rev Pharmacol Toxicol 2019. https://doi.org/10. 1146/annurev-pharmtox-010818-021059.

12. Parry GJ, Rodrigues CMP, Aranha MM, Hilbert SJ, Davey C, Kelkar P, et al. Safety, tolerability, and cerebrospinal fluid penetration of ursodeoxycholic Acid in patients with amyotrophic lateral sclerosis. Clin Neuropharmacol 2010;33:17-21. https://doi.org/10. 1097/WNF.0b013e3181c47569.

13. Daruich A, Le Rouzic Q, Jonet L, Naud M-C, Kowalczuk L, Pournaras J-A, et al. Iron is neurotoxic in retinal detachment and transferrin confers neuroprotection. Sci Adv 2019;5:eaau9940. https://doi.org/10.1126/sciadv.aau9940.

14. Couturier A, Bousquet E, Zhao M, Naud M-C, Klein C, Jonet L, et al. Anti-vascular endothelial growth factor acts on retinal microglia/macrophage activation in a rat model of ocular inflammation. Mol Vis 2014;20:908-20.

15. Sanges D, Comitato A, Tammaro R, Marigo V. Apoptosis in retinal degeneration involves cross-talk between apoptosis-inducing factor (AIF) and caspase-12 and is blocked by calpain inhibitors. Proc Natl Acad Sci U S A 2006;103:17366-71. https://doi.org/10. 1073/pnas.0606276103.
16. Brylev LV, Zakharova MN, Zavalishin IA, Gulyaeva NV. Disruption of blood-brain barrier in amyotrophic lateral sclerosis: an update. Neurochem J 2012;6:64-70. https://doi.org/10.1134/ S1819712411040064.

17. Torriglia A, Valamanesh F, Behar-Cohen F. On the retinal toxicity of intraocular glucocorticoids. Biochem Pharmacol 2010;80:187886. https://doi.org/10.1016/j.bcp.2010.07.012.

18. Kowalczuk L, Matet A, Dor M, Bararpour N, Daruich A, Dirani A, et al. Proteome and Metabolome of Subretinal Fluid in Central Serous Chorioretinopathy and Rhegmatogenous Retinal Detachment: A Pilot Case Study. Transl Vis Sci Technol 2018;7: 3. https://doi.org/10.1167/tvst.7.1.3.

19. Quintyn J-C, Brasseur G. Subretinal fluid in primary rhegmatogenous retinal detachment: physiopathology and composition. Surv Ophthalmol 2004;49:96-108. https://doi.org/10.1016/ j.survophthal.2003.10.003.

20. Mitry D, Awan MA, Borooah S, Syrogiannis A, Lim-Fat C, Campbell $\mathrm{H}$, et al. Long-term visual acuity and the duration of macular detachment: findings from a prospective populationbased study. Br J Ophthalmol 2013;97:149-52. https://doi.org/10. 1136/bjophthalmol-2012-302330.

21. Liu Z, Liu J, Wang S, Liu S, Zhao Y. Neuronal uptake of serum albumin is associated with neuron damage during the development of epilepsy. Exp Ther Med 2016;12:695-701. https://doi.org/10. 3892/etm.2016.3397.

22. Potic J, Mbefo M, Berger A, Nicolas M, Wanner D, Kostic C, et al. An in vitro Model of Human Retinal Detachment Reveals Successive Death Pathway Activations. Front Neurosci 2020;14: 571293. https://doi.org/10.3389/fnins.2020.571293.

23. Gaspar JM, Martins A, Cruz R, Rodrigues CMP, Ambrósio AF, Santiago AR. Tauroursodeoxycholic acid protects retinal neural cells from cell death induced by prolonged exposure to elevated glucose. Neuroscience 2013;253:380-8. https://doi.org/10.1016/j. neuroscience.2013.08.053.

24. Tao Y, He M, Yang Q, Ma Z, Qu Y, Chen W, et al. Systemic taurine treatment provides neuroprotection against retinal photoreceptor degeneration and visual function impairments. Drug Des Devel Ther 2019;13:2689-702. https://doi.org/10.2147/DDDT. S194169.

25. Beli E, Yan Y, Moldovan L, Vieira CP, Gao R, Duan Y, et al. Restructuring of the Gut Microbiome by Intermittent Fasting Prevents Retinopathy and Prolongs Survival in $\mathrm{db} / \mathrm{db}$ Mice. Diabetes 2018;67:1867-79. https://doi.org/10.2337/db18-0158.

26. Li L, Du G, Wang D, Zhou J, Jiang G, Jiang H. Overexpression of Heme Oxygenase-1 in Mesenchymal Stem Cells Augments Their Protection on Retinal Cells In Vitro and Attenuates Retinal Ischemia/Reperfusion Injury In Vivo against Oxidative Stress. Stem Cells Int 2017;2017:4985323. https://doi.org/10.1155/2017/ 4985323.

27. Himori N, Maruyama K, Yamamoto K, Yasuda M, Ryu M, Omodaka K, et al. Critical neuroprotective roles of heme oxygenase-1 induction against axonal injury-induced retinal ganglion cell death. J Neurosci Res 2014;92:1134-42. https://doi.org/ 10.1002/jnr.23398.

28. Fan $\mathrm{J}, \mathrm{Xu} \mathrm{G}$, Jiang T, Qin Y. Pharmacologic induction of heme oxygenase-1 plays a protective role in diabetic retinopathy in rats. Invest Ophthalmol Vis Sci 2012;53:6541-56. https://doi.org/10. 1167/iovs.11-9241.

29. Shyong M-P, Lee F-L, Hen W-H, Kuo P-C, Wu A-C, Cheng H-C, et al. Viral delivery of heme oxygenase-1 attenuates photoreceptor apoptosis in an experimental model of retinal detachment. Vis Res 2008;48:2394-402. https://doi.org/10.1016/j.visres.2008.07.017.

30. Ito $\mathrm{Y}$, Tanaka $\mathrm{H}, \mathrm{Hara} \mathrm{H}$. The potential roles of metallothionein as a therapeutic target for cerebral ischemia and retinal diseases. Curr Pharm Biotechnol 2013;14:400-7. https://doi.org/10.2174/ 1389201011314040003. 
31. Saul KE, Koke JR, García DM. Activating transcription factor 3 (ATF3) expression in the neural retina and optic nerve of zebrafish during optic nerve regeneration. Comp Biochem Physiol, Part A Mol Integr Physiol 2010;155:172-82. https://doi.org/10.1016/j. cbpa.2009.10.042.

32. Gey M, Wanner R, Schilling C, Pedro MT, Sinske D, Knöll B. Atf3 mutant mice show reduced axon regeneration and impaired regeneration-associated gene induction after peripheral nerve injury. Open Biol 2016;6. https://doi.org/10.1098/rsob.160091.

33. Ye D, Shi Y, Xu Y, Huang J. PACAP Attenuates Optic Nerve Crush-Induced Retinal Ganglion Cell Apoptosis Via Activation of the CREB-Bcl-2 Pathway. J Mol Neurosci 2019;68:475-84. https:// doi.org/10.1007/s12031-019-01309-9.

34. Vaczy A, Kovari P, Kovacs K, Farkas K, Szabo E, Kvarik T, et al. Protective Role of Endogenous PACAP in Inflammation-induced Retinal Degeneration. Curr Pharm Des 2018;24:3534-42. https:// doi.org/10.2174/1381612824666180924141407.

35. Bramall AN, Szego MJ, Pacione LR, Chang I, Diez E, D'OrleansJuste P, et al. Endothelin-2-mediated protection of mutant photoreceptors in inherited photoreceptor degeneration. PLoS One 2013;8: e58023. https://doi.org/10.1371/journal.pone.0058023.

36. Braunger BM, Ohlmann A, Koch M, Tanimoto N, Volz C, Yang Y, et al. Constitutive overexpression of Norrin activates $\mathrm{Wnt} / \beta$-catenin and endothelin-2 signaling to protect photoreceptors from light damage. Neurobiol Dis 2013;50:1-12. https://doi.org/10.1016/j. nbd.2012.09.008.

37. Lakowski J, Gonzalez-Cordero A, West EL, Han Y-T, Welby E, Naeem A, et al. Transplantation of Photoreceptor Precursors Isolated via a Cell Surface Biomarker Panel From Embryonic Stem Cell-Derived Self-Forming Retina. Stem Cells 2015;33: 2469-82. https://doi.org/10.1002/stem.2051.

38. Yasuda M, Tanaka Y, Ryu M, Tsuda S, Nakazawa T. RNA sequence reveals mouse retinal transcriptome changes early after axonal injury. PLoS One 2014;9:e93258. https://doi.org/10.1371/ journal.pone.0093258.

39. Lee YS, Amadi-Obi A, Yu C-R, Egwuagu CE. Retinal cells suppress intraocular inflammation (uveitis) through production of interleukin-27 and interleukin-10. Immunology 2011;132:492502. https://doi.org/10.1111/j.1365-2567.2010.03379.x.

40. Amadi-Obi A, Yu C-R, Dambuza I, Kim S-H, Marrero B, Egwuagu CE. Interleukin 27 induces the expression of complement factor $\mathrm{H}$ (CFH) in the retina. PLoS One 2012;7:e45801. https://doi.org/10. 1371/journal.pone.0045801.

Publisher's Note Springer Nature remains neutral with regard to jurisdictional claims in published maps and institutional affiliations. 